\title{
Serotonin 2C Receptor Alternative Splicing in a Rat Model of Orofacial Neuropathic Pain
}

\author{
Aya Nakae $^{1 *}$, Kunihiro Nakai ${ }^{2}$, Tatsuya Tanaka ${ }^{3}$, Ko Hosokawa $^{2}$, Takashi Mashimo \\ ${ }^{1}$ Department of Anesthesiology \& Intensive Care, Graduate School of Medicine, Osaka University, Osaka, Japan; ${ }^{2}$ Department of \\ Plastic Surgery, Graduate School of Medicine, Osaka University, Osaka, Japan; ${ }^{3}$ Center for Medical Research and Education, Gradu- \\ ate School of Medicine, Osaka University, Osaka, Japan. \\ Email: *anakae@anes.med.osaka-u.ac.jp
}

Received December $11^{\text {th }}, 2011$; revised January $20^{\text {th }}, 2012$; accepted February $12^{\text {th }}, 2012$

\begin{abstract}
Abnormal serotonin 2C receptor (5HTR2C) alternative splicing and RNA editing are involved in the etiology of pain disorders. Functional 5HTR2C can only be generated when alternative exon $\mathrm{Vb}$ is included within the mRNA; the small nucleolar RNA RBII-52 is complementary to exon $\mathrm{Vb}$ and promotes its inclusion. The expression of HBII-52 (the human equivalent of RBII-52) is reduced in Prader-Willi syndrome, patients of which have a high pain threshold. Here, we measured the pain threshold in a rat model of orofacial neuropathic pain and related it to the expression levels of wild-type and variant 5HTR2C and RBII-52. We generated an infraorbital nerve loose ligation model of neuropathic pain in rats and measured the pain threshold of the animals using mechanical stimulation with von Frey filaments. We then sacrificed the animals and examined the RNA levels of 5HTR2C and RBII-52 in the cervical spinal cord by real-time PCR. On post-injury day 28, pain threshold values in injured rats were significantly lower than in sham-operated or naïve animals. The levels of total and exon Vb-skipped 5HTR2C mRNA were significantly lower in injured rats than in that sham-operated or naïve rats, and the ratio of exon Vb-skipped 5HTR2C to total 5HTR2C was significantly higher. There were no significant differences in RBII-52 expression among the groups. Our data suggest that neuropathic pain induces serotonergic dysfunction mediated by 5HTR2C alternative splicing. 5HTR2C might be subject to complicated and fine regulation both by RNA editing and by alternative splicing.
\end{abstract}

Keywords: Orofacial Neuropathic Pain; Prader-Willi Syndrome; Serotonin 2C Receptor; Alternative Splicing; SnoRNA; RBII-52; RNA Editing; Spinal Cord

\section{Introduction}

Prader-Willi syndrome (PWS) is a neurodevelopmental disorder caused in the majority of cases by deletion of the paternally derived chromosome 15 (del15q11-13) or maternal uniparental disomy of chromosome 15 [1,2]. The clinical features of PWS include hyperphagia, obesity, body temperature instability, hypogonadism, and altered growth hormone secretion $[1,3]$, suggesting hypothalamic dysfunction. Furthermore, because of hypothalamic-pituitary involvement, PWS patients have a high pain threshold [4]; this is one of the supportive criteria for the diagnosis of PWS [5].

The descending serotonin (5HT) pathway normally inhibits signaling from noxious stimuli. Dysfunction in this pathway can result in hypersensitivity to pain and a low pain threshold [6] and is a common neurological abnormality implicated in the etiology of pain disorders [6,7]. The 5HT 2C receptor (5HTR2C) plays an important role

\footnotetext{
${ }^{*}$ Corresponding author.
}

in spinal inhibition of trigeminal neuropathic pain [8].

5HTR2C is a G-protein-coupled receptor whose premRNA is a substrate for base modification that, via hydrolytic deamination of adenosines, yields inosines [9]. Five adenosines (present at editing sites A-E Figure 1(a)), located within a sequence encoding the putative second intracellular domain of 5HTR2C, can be converted to inosines. This editing can alter the coding potential of three triplet codons and permits the generation of 24 different receptor isoforms ranging from the unedited Ile156Asn158Ile160 (INI) to the fully edited Val156Gly158Val160 (VGV). Receptors resulting from fully edited transcripts, and from partially edited transcripts that include editing of at least the $\mathrm{E}$ site or of both the $\mathrm{E}$ and $\mathrm{C}$ sites, differ from non-edited receptors by their lower affinity for serotonin and, consequently, their reduced ability to activate G-protein [9-11]. Such differences in G-protein coupling efficiency associated with RNA editing can be accounted for by differences in the conformational properties of the second intracellular loop in 5HTR2C [12]. In effect, over $60 \%$ of 


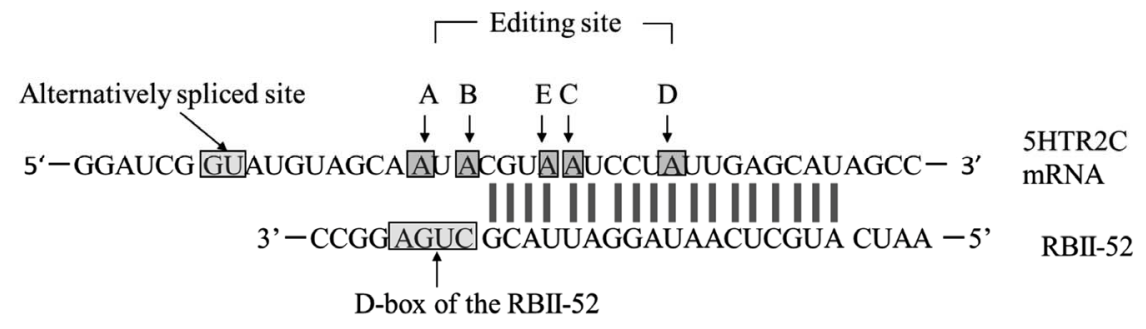

(a)
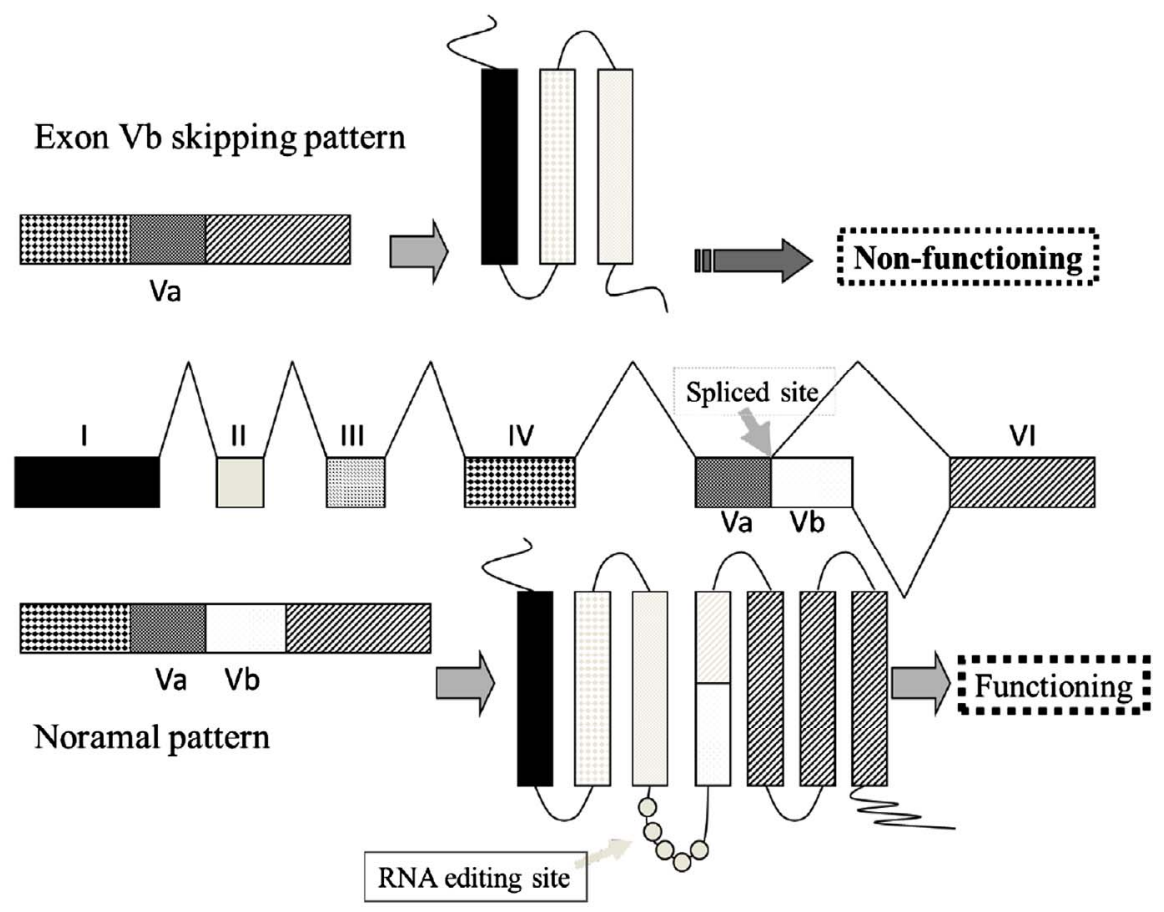

(b)

Figure 1. (a) Complementarity between snoRNA RBII-52 and 5HTR2C. The upper sequence shows the rat 5HTR2C mRNA; the lower sequence shows the snoRNA RBII-52. Arrows indicate the editing sites A-E where A-to-I editing can occur. The alternative splice donor site is close to the editing sites. Gray lines indicate the 18-nucleotide complementarity between RBII52 and 5HTR2C; (b) Structure of the rat5HTR2C splicing variants. Alternate usage of exon Vb gives rise to two mRNA isoforms that are indicated schematically. Exon Vb-skipping results in a truncated receptor shown schematically above the mRNA.

all 5HTR2C mRNAs encode receptors with reduced constitutive and agonist-stimulated activity. Previously, in a rat model of neuropathic pain, we speculated that modulation of 5HTR2C mRNA editing could be an adaptive mechanism in response to nociceptive stimuli $[13,14]$.

As an additional layer of complexity, the 5HTR2C mRNA is also subject to alternative splicing. The normal intron V 5' splice site, which is contained within the stemloop region containing the five editing sites [9], is flanked by an alternative splice site (Figure 1(b)), which can give rise to isoforms containing exon $\mathrm{Va}$ with or without exon $\mathrm{Vb}$. Exon $\mathrm{Vb}$ encodes the second intracellular loop of the receptor, which is crucial for G-protein binding. Skipping of exon $\mathrm{Vb}$ causes a frameshift, resulting in a receptor that is truncated after the third transmembrane domain [11]. Moreover, RNA editing modulates splice site selection: the strongest predictor of a Vb-skipping splice pattern, is unedited mRNA. This unedited mRNA encodes the INI isoform, which exhibits constitutive activity [10,15]. Consequently, when editing is inefficient, a pattern of increased Vb-skipping (presumably resulting in a proportional increase in inactive receptors) may act as a control mechanism to lessen the biosynthesis of the INI isoform and thereby limit constitutive activity [16].

Small non-coding nucleolar RNAs (snoRNAs) are nonprotein-coding RNAs that are 60 to 300 nucleotides long. They guide 2'-O-methylation and pseudo-uridylation in ribosomal RNAs, small nuclear RNAs, and tRNAs [17]. The evolutionarily conserved snoRNA HBII-52 is a key regulator of post-transcriptional modification of the 5HTR2C pre-mRNA [18] and is encoded within the PWS-critical region on chromosome 15 . RBII-52, the rat equivalent of 
human HBII-52, is a brain-specific C/D box snoRNA whose antisense element is complementary to the alternatively spliced exon $\mathrm{Vb}$ of 5HTR2C and which promotes the inclusion of this exon. Interestingly, HBII-52 plays an important role in PWS. Specific behavioral effects were observed in a mouse model of PWS lacking expression of the mouse equivalent, MBII-52 [18].

In this study, we speculated that the allodynia of rats that had undergone infraorbital nerve loose ligation (ION-LL) and consequently demonstrated pain-related behavior, would be affected by 5HTR2C mRNA editing efficiency and alternative splicing in the spinal cord, and that any changes would be related to RBII-52. To test this hypothesis, we designed an ION-LL rat model and analyzed alternative splicing of 5HTR2C and RBII-52 expression in the spinal cord.

\section{Materials and Methods}

All surgical and experimental protocols in this study were reviewed and approved by the Institutional Animal Care and Use Committee of Osaka University and were carried out according to the National Institutes of Health guidelines. The general treatment of animals conformed to the Guidelines of the International Association for the Study of Pain [19]. In particular, the duration of the experiments and the number of experimental animals (total $n=45$ ) were minimized.

\subsection{Experimental Animals}

Each group consisted of 10 male Sprague-Dawley (SD) rats of body weight $170 \mathrm{~g}-230 \mathrm{~g}$ at the time of surgery. Rats were housed four to a cage with free access to chow and water at $22^{\circ} \mathrm{C} \pm 2^{\circ} \mathrm{C}$ under a $12 \mathrm{~h}$ light-dark cycle (illumination 08:00 - 20:00). The animals were allowed at least 1 week to settle into their housing conditions before surgery.

\subsection{Surgical Procedures}

Under direct manipulation while viewing through a surgical microscope, we applied unilateral chronic constriction injuries to the right ION, essentially according to the method described by Vos et al. [20]. In brief, the animals were anesthetized with sodium pentobarbital (Nembutal; $60 \mathrm{mg} / \mathrm{kg}$ i.p.). A midline scalp incision was made, exposing the skull and nasal bone. The edge of the orbit, formed by the maxillary, frontal, lacrimal, and zygomatic bones, was exposed by dissection. Then the ION was dissected free from its most rostral location in the orbital cavity, just caudal to the infraorbital foramen. Two nylon (5-0) ligatures (2 mm apart) were tied loosely around the ION. To obtain the desired degree of constriction, the criterion formulated by Bennet and Xie [21] was applied: the ligature reduced the diameter of the nerve by a just noticeable amount and retarded, but did not interrupt, epineural circulation through the superficial vasculature. The scalp incision was closed in layers using nylon sutures $(5-0)$. Sham operations were performed in the same way, but the two nylon ligatures were more loosely tied around the ION. After surgery, the rats were allowed to recover in a warmed cage in which water and chow were easily accessible.

\subsection{Behavioral Testing}

Behavioral tests were carried out 3 days before and 7, 14, 21 , and 28 days after surgery. All experiments were carried out in a quiet room generally between 09:00 and 16:00. Each rat was placed alone in a plastic cage $(25 \mathrm{~cm}$ $\times 40 \mathrm{~cm} \times 18 \mathrm{~cm}$ ) with bedding, and left for $30 \mathrm{~min}$ of accommodation. To determine pain hypersensitivity to mechanical stimulation, von Frey filaments of increasing resilience (bending force 0.16, 0.4, 0.6, 1.0, 1.4, 2.0, 4.0, $6.0,8.0,10.0$, and $15.0 \mathrm{~g}$ ) were sequentially applied from above to the ION territory, near the center of the vibrissal pad, on the hairy skin surrounding the mystacial vibrissae. Each von Frey filament was applied five times to the same region at approximately $1 \mathrm{~s}$ intervals. Head withdrawal or touching or scratching of the facial region upon application of a von Frey filament was recorded as a positive pain response. The response threshold was defined as the lowest-force filament application that prompted at least three positive responses in five trials. If an animal made fewer than three pain responses to any of the tested filaments, $15 \mathrm{~g}$ was taken to be the threshold.

\subsection{Total RNA Extraction, Reverse Transcription, and Real-Time PCR}

On post-injury day 28 , the animals were sacrificed by decapitation. Cervical spinal cord tissue samples were then taken from each animal and were retained in RNAlater solution (Ambion, Inc., Tokyo, Japan). Following the manufacturer's protocol, total RNA was extracted using an RNeasy Lipid Tissue Mini Kit (Qiagen, Tokyo, Japan). Ten micrograms of total RNA were then used for firststrand cDNA synthesis by Thermoscript reverse transcriptase and random hexamers (Invitrogen, Tokyo, Japan). The amounts of total 5HTR2C, and exon Vb-skipped 5HTR2C were evaluated, according to the manufacturer's protocol, by quantitative real-time RT-PCR with an ABI PRISM 7900HT (Applied Biosystems, Foster City, CA) and TaqMan probes designed and supplied by Applied Biosystems. The primer and probe sequences were as follows: total 5HTR2C (designed in exons 1 and 2): (forward);

5'-CGAGTCCGTTTCTCGTCTAGCT-3', (reverse); 5'-TTGGCCTATGCTTGCAGGTA-3', and (TaqMan MGBprobe);

5'-CGGGTTGTCAACTATT-3';

Vb-skipped 5'-HTR2C: (forward); 


\section{5'-GCTTGCTATTCTTTATGATTAT-GTCTGG-3', (reverse); 5'-GGAACTGAAACTCCGGT-CCA-3', and (TaqMan MGBprobe); \\ 5'-CATGATGGACGCAGTTG-3'; RBII-52: (forward); 5'-GGGTCAATGATGACAACATTAAGTCA-3', (reverse); 5'-GGGCCTCAGCGTAATCCTATT-3', and (TaqMan MGBprobe); \\ 5'-AGAACAGAATGATGACATAAA-3'.}

All reactions were performed in triplicate. The data were normalized to those obtained for the internal control glyceraldehyde 3-phosphate dehydrogenase (GAPDH), and averaged for each group.

\subsection{Data Analysis}

Behavioral data for the time course were analyzed using two-way analysis of variance, and statistical differences were examined post-hoc using the Tukey-Kramer multiple-comparison test. The mRNA data were analyzed using the Student's $t$-test after multiple comparisons by analysis of variance $(p<0.05)$. All data are presented as the mean \pm standard error of the mean.

\section{Results}

\subsection{Behavioral Responses to ION-LL}

On post-injury days 14 and 28, ION-LL was significantly correlated with reflex withdrawal to punctate mechanical stimulation. Pain thresholds in the territory affected by ION-LL were significantly lower for injured rats than for sham-operated or naïve animals (Figure 2(a)).

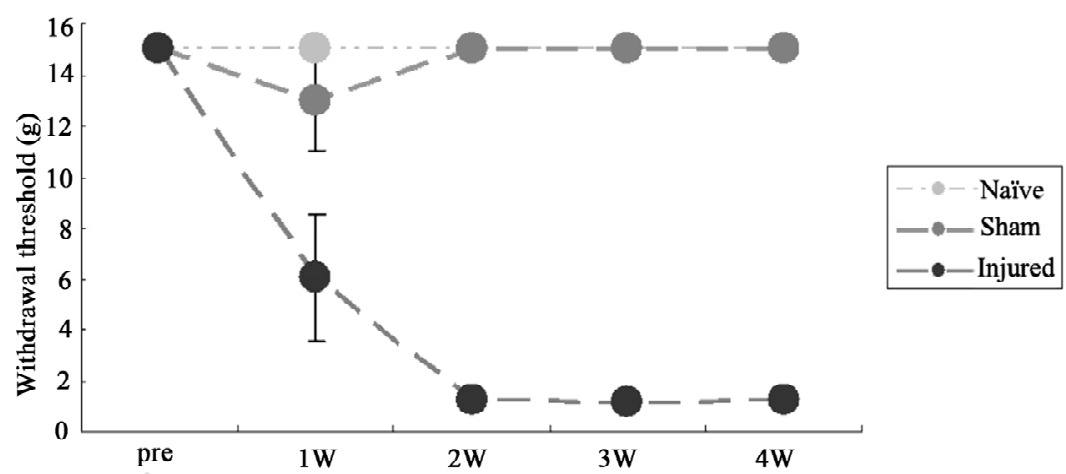

(a)
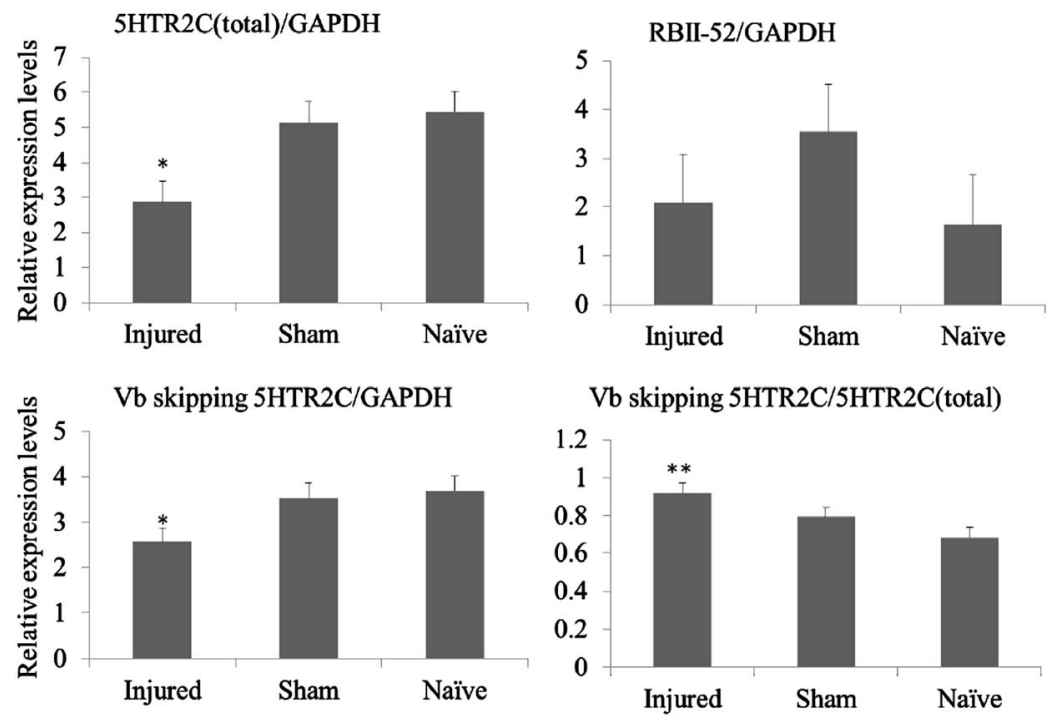

(b)

Figure 2. (a) Behavioral responses to infraorbital nerve loose ligation (ION-LL). On post-injury day 28, ION-LLwas significantly correlated with reflex withdrawal from mechanical stimulation using von Frey filaments. Pain threshold values from the infraorbital nerve territory in ION-LL-injured rats were significantly lower than those in sham-operatedor naïve animals. The data represent mean \pm standard error of the mean often rats in each group; (b) Total 5HTR2C, exon Vb-skipped 5HTR2C, and RBII-52 expression in the spinal cord. The expression of total 5HTR2C and exon Vb-skipped 5HTR2C in samples from injured rats was lower than that in samples from naïve or sham-operated rats $\left({ }^{*} p<0.05\right)$. The ratio of alternatively spliced 5HTR2C to total 5HTR2C was higher in samples from injured rats than in samples from sham-operatedor naïve rats $(* * p<0.01)$. There was no significant difference in RBII-52 expression among the group. 


\subsection{HTR2C Expression Levels and Alternative Splicing in the Spinal Cord}

The mRNA levels of total 5HTR2C and exon Vb-skipped 5HTR2C were significantly lower in samples from injured rats than in samples from sham-operated or naïve rats ( $p$ $<0.05$; Figure 2(b)). In addition, the ratio of exon Vbskipp-ed 5HTR2C to total 5HTR2C was significantly higher in samples from injured rats than in those from shamoperated or naïve rats ( $p<0.01$; Figure 2(b)).

\subsection{Correlation between RBII-52 Expression Level and Expression of the Exon Vb-Skipped Splice Variant}

There was no significant difference in RBII-52 expression among the groups (Figure 2(b)), and no correlation between RBII-52 expression and the expression level of the exon Vb-skipped splice variant.

\section{Discussion}

Using a model of orofacial neuropathic pain, we investigated the relationship between pain sensitivity and alternative splicing of 5HTR2C pre-mRNA or expression of the snoRNA RBII-52. Rats injured by ION-LL exhibited greater pain sensitivity on post-injury day 28 than uninjured rats, as attested by lower thresholds to mechanical stimulation using von Frey filaments. On post-injury day 28, total 5HTR2C expression was significantly lower in injured rats than in sham-operated or naïve rats, and the ratio of the exon Vb-skipped splicing variant (encoding a non-functional receptor) to total 5HTR2C was significantly increased.

PWS is associated with reduced sensitivity to painful and thermal stimuli [4], which is interpreted to be a consequence of hypothalamic-pituitary involvement. However, the altered pain perception in this syndrome remains largely unexplained. HBII-52, a snoRNA whose absence causes behavioral changes similar to PWS in mice [18], is located within the PWS-critical region on chromosome 15. Among the many targets of HBII-52 [22], only orexin and 5HTR2C are considered to potentially affect pain-related behavior. Moreover, our previous 5HTR2C RNA editing results were similar to data derived from PWS patients [13]. Thus, we attempted to clarify RBII-52 expression and the relationship between RBII-52 and 5HTR2C alternative splicing in a model of orofacial neuropathic pain.

Our study revealed a relationship between 5HTR2C splicing and pain-related behavior. We tried to clarify the relationship between 5HTR2C splicing and the snoRNA RBII-52, but the study design only allowed us to the evaluate the relative expression levels of total 5HTR2C and exon Vb-skipped 5HTR2C which is the limitation of the quantitative real time PCR because efficiency of PCR is not completely same. We were unable to measure the real ratio between exon Vb-skipped 5HTR2C and total 5HTR2C; therefore, we were unable to assess the influence of alternative splicing. The spinal cords of injured rats demonstrated a significant increase in the ratio of exon Vbskipped 5HTR2C to total 5HTR2C. This finding suggests that 5HTR2C function declined because of alternative splicing induced in the presence of noxious stimuli. In other words, in the presence of neuropathic pain, the balance of splice variants shifted toward exon Vb-skipped 5HTR2C transcripts that encode a non-functional protein.

In our previous study of 5HTR2C RNA editing [13], we found a greater presence of 5HTR2C isoforms with increased basal activity and fewer less active isoforms in the orofacial neuropathic pain model. Our current findings convey the opposite. In our previous study, the RNA editing efficiency at sites A and B of 5HTR2C was significantly lower in ipsilateral injured spinal cord [13]. These changes are similar to those found in brain samples of post-mortem PWS patients. However, in this study, there was no significant decrease in RBII-52 expression in injured rats. In a mouse model of PWS lacking expression of MBII-52, increased levels of 5HTR2C pre-mRNA editing, but not of alternative splicing, were observed in the brain [18]. These results are contradictory to ours that showed reduced editing at sites A and B. Thus, the lack of RBII-52 might not directly influence RNA editing efficiency and alternative splicing; other factors may be required.

In our study, the influence of non-functional 5HTR2C was unclear, because the proportion of spliced non-functional receptor could not be analyzed. In our previous study, the proportion of non-edited 5HTR2C was about half of the total 5HTR2C. Furthermore, the non-edited 5HTR2C exhibits greater constitutive activity (5HTR2C can exist in a conformation that mimics an active state even in the absence of an agonist). Thus, RNA editing efficiency might be considered to exert a greater effect than alternative splicing on serotonin signal transduction in our model of orofacial neuropathic pain. However, further studies are required to confirm the influence of alternative splicing of 5HTR2C.

In conclusion, in our rat model of orofacial neuropathic pain, 5HTR2C expression in the spinal cord was inhibited and the ratio of the exon Vb-skipped variant (which encodes a non-functional protein) to total 5HTR2C was increased. In other words, 5HTR2C function was negatively regulated by alternative splicing in the neuropathic pain model. Our current findings do not agree with those of our previous 5HTR2C RNA editing study. The modulation of 5HTR2C activity in response to noxious stimuli such as neuropathic pain might be capable of much finer tuning than we previously imagined.

\section{Acknowledgements}

This work was supported by a Grant-in-Aid (No. 20791070) from the Ministry of Education, Culture, Sports, Science, 
and Technology, Japan; and by Special Coordination Funds for Promoting Science and Technology from the Ministry of Education, Culture, Sports, Science, and Technology for the Osaka University Program for the Support of Networking among Present and Future Women Researchers. The authors thank Mari Yoshida, Akiko Mikami, and Yoko Ishida for excellent technical assistance.

\section{REFERENCES}

[1] S. B. Assidy and D. J. Driscoll, "Prader-Willi Syndrome," European Journal of Human Genetics, Vol. 17, No. 1, 2009, pp. 3-13. doi:10.1038/ejhg.2008.165

[2] R. D. Nicholls, S. Sautoh and B. Horsthemke, "Imprinting in Prader-Willi and Angelman Syndromes," Trends in Genetics, Vol. 14, No. 5, 1998, pp. 194-200. doi:10.1016/S0168-9525(98)01432-2

[3] M. S. Williams, B. L. Rooney, J. Williams, K. Josephson and R. Pauli, "Investigation of Thermoregulatory Characteristics in Patients with Prader-Willi Syndrome," American Journal of Medical Genetics, Vol. 49, No. 3, 1994, pp. 302-307. doi:10.1002/ajmg.1320490312

[4] L. Priano, G. Miscio, G. Grugni, E. Milano, S. Baudo, L. Sellitti, R. Picconi and A. Mauro, "On the Origin of Sensory Impairment and Altered Pain Perception in PraderWilli Syndrome: A Nuerological Study,” European Journal of Pain, Vol. 13, No. 8, pp. 829-835. doi:10.1016/j.ejpain.2008.09.011

[5] V. A. Holm, S. B. Cassidy, M. G. Butler, J. M. Hanchett, L. R. Greenswag, B. Y. Whitman and F. Greenberg, "PraderWilli Syndrome: Consensus Diagnostic Criteria,” Pediatrics, Vol. 91, No. 2, 1993, pp. 398-402

[6] S. Stahl and M. Briley, "Understanding Pain in Depression," Human Psychopharmacology, No. S1, 2004, pp. S19S13.

[7] A. I. Basbaum and H. L. Fields, "Endogenous Pain Control Systems: Brainstem Spinal Pathways and Endorphin Circuitry,” Annual Review of Neuroscience, Vol. 7, 1984, pp. 309-338. doi:10.1146/annurev.ne.07.030184.001521

[8] K. Nakai, A. Nakae, S. Aba, T. Mashimo and K. Ueda, "5-HT2C Receptor Agonists Attenuate Pain-Related Behaviour in a Rat Model of Trigeminal Neuropathic Pain," European Journal of Pain, Vol. 14, No. 10, 2010, pp. 999-1006. doi:10.1016/j.ejpain.2010.04.008

[9] C. M. Burns, H. Chu, S. M. Rueter, L. K. Hutchinson, H. Canton, E. Sander-Bush and R. B. Emeson, "Regulation of Serotonin-2C Receptor G-Protein Coupling by RNA Editing,” Nature, Vol. 387, No. 6630, 1997, pp. 303-308. doi:10.1038/387303a0

[10] C. M. Niswender, S. C. Copeland, K. Herrick-Davis, R. B. Emeson and E. Sander-Bush, "RNA Editing of the $\mathrm{Hu}-$ man Serotonin 5-Hydroxytryptamine 2C Receptor Silences Consititutive Activity,” Journal of Biological Chemistry, Vol. 274, No. 14, 1999, pp. 9472-9478. doi:10.1074/jbc.274.14.9472

[11] Q. Wang, P. J. O’Brien, C. X. Chen, D. S. Cho, J. M., Murray and K. Nishikura, "Altered G Protein-Coupling Functions of RNA Editing Isoform and Splicing Variant
Serotonin 2C Receptors,” Journal of Neurochemistry, Vol. 74, No. 3, 2000, pp. 1290-300. doi:10.1046/j.1471-4159.2000.741290.x

[12] I. Visiers, S. A. Hassan and H. Weinstein, "Differences in Conformational Properties of Second Intracellular Loop (IL2) in 5HT(2C) Receptors Modified by RNA Editing ca Account for G Protein Coupling Efficiency,” Protein Engineering, Vol. 14, No. 6, 2001, pp. 409-414. doi:10.1093/protein/14.6.409

[13] A. Nakae, K. Nakai, T. Tanaka, S. Hagihira, M. Shibata, K. Ueda and T. Mashimo, "The Role of RNA Editing of the Serotonin 2C Receptor in a Rat Model of Oro-Facial Neuropathic Pain,” European Journal of Neuroscience, Vol. 27, No. 9, 2008, pp. 2373-2379. doi:10.1111/j.1460-9568.2008.06205.x

[14] A. Nakae, K. Nakai, T. Tanaka, M. Takashina, S. Hagihira, M. Shibata, K. Ueda and T. Mashimo, "Serotonin 2C Receptor mRNA Editing in Neuropathic Pain Model,” Neuroscience Research, Vol. 60, No. 2, 2008, pp. 228231. doi:10.1016/j.neures.2007.10.004

[15] K. Herrick-Davis, E. Grinde and C. M. Niswender, "Serotonin 5-HT2C Receptor RNA Editing Alters Receptor Basal Activity: Implications for Serotonergic Signal Transdaction,” Journal of Neurochemistry, Vol. 73, No. 4, 1999, pp. 1711-1717. doi:10.1046/j.1471-4159.1999.731711.x

[16] R. Flomen, J. Knight, P. Sham, R. Kerwin and A. Makoff, "Evidence That RNA Editing modulates Splice Site Selection in the 5-HT2C Receptor Gene," Nucleic Acids Research, Vol. 32, No. 7, 2004, pp. 2113-2122. doi:10.1093/nar/gkh536

[17] T. Kiss, "Small Nucleolar RNAs: An Abundant Group of Noncoding RNAs with Doverse Cellular Functions," Cell, Vol. 109, No. 2, 2002, pp. 145-148. doi:10.1016/S0092-8674(02)00718-3

[18] C. M. Doe, D. Relkovic, A. S. Garfield, J. W. Dalley, D. E. Theobald, T. Humby, L. S. Wilkinson and A. R. Isles, "Loss of the Imprinted snoRNA mbii-52 Leads to Increased 5htr2c Pre-RNA Editing and Altered 5HT2cR Mediated Behaviour," Human Molecular Genetics, Vol. 18, No. 12, 2009, pp. 2140-2158. doi:10.1093/hmg/ddp137

[19] M. Zimmermann, "Ethical Guidelines for Investigations of Experimental Pain in Conscious Animals,” Pain, Vol. 16, No. 2, 1983, pp. 109-110. doi:10.1016/0304-3959(83)90201-4

[20] B. P. Vos, A. M. Strassman and R. J. Maciewicz, "Behavioral Evidence of Trigeminal Neuropathic Pain Following Chronic Constiriction Injury to the Rat's Infraorbital Nerve,” Journal of Neuroscience, Vol. 14, 1994, pp. 2708-2723

[21] G. J. Bennett and Y. K. Xie, “A Peripheral Mononeuropathy in Rat That Produces Disorders of Pain Sensation Like Those Seen in Man,” Pain, Vol. 33, No. 1, 1984, pp. 87-107. doi:10.1016/0304-3959(88)90209-6

[22] S. Kishore, "The snoRNA HBII-52 Absent in PraderWilli Syndrome Patients Regulates the Alternative Splicing of the Serotonin Receptor 5-HT2cR,” 1984. http://www.stamms-labnet/pdfs/theses/Kishore_Thesis.pdf 